\title{
Prediction and identification of epitopes in the Echinococcus multilocularis thrombospondin 3 antigen
}

\author{
Ming-Quan Pang ${ }^{\mathrm{a}, \mathrm{b}, \mathrm{c}, 1}$, Yue-Qing Lu ${ }^{\mathrm{a}, \mathrm{c}, 1}$, Feng Tang ${ }^{\mathrm{b}, \mathrm{c}}$, Hai-Jiu Wang ${ }^{\mathrm{a}, \mathrm{c}}$, Ying Zhou ${ }^{\mathrm{a}, \mathrm{c}}$, \\ $\mathrm{Li} \mathrm{Ren}^{\mathrm{a}, \mathrm{c}}$, Run-Le Li ${ }^{\mathrm{b}, \mathrm{c}}$, Hu Zhou ${ }^{\mathrm{a}, \mathrm{c}}$, Chen-Fei Wan ${ }^{\mathrm{b}, \mathrm{c}}$, Chuan-Chuan Liu ${ }^{\mathrm{a}, \mathrm{b}, \mathrm{c}}$, \\ Dawa Luosang ${ }^{\mathrm{b}, \mathrm{c}}$, Cairang Yangdan ${ }^{\mathrm{a}, \mathrm{c}}$ and Hai-Ning Fan ${ }^{\mathrm{a}, \mathrm{c}, *}$ \\ ${ }^{a}$ Department of Hepatopancreatobiliary Surgery, Affiliated Hospital of Qinghai University, Xining, \\ Qinghai, China \\ ${ }^{\mathrm{b}}$ Research Center for High Altitude Medical Sciences, Qinghai University School of Medicine, Xining, \\ Qinghai, China \\ ${ }^{\mathrm{C}}$ Qinghai Provincial Research Key Laboratory for Hydatid, Xining, Qinghai, China
}

Received 25 March 2021

Accepted 22 November 2021

\begin{abstract}
.
BACKGROUND: Alveolar echinococcosis is an epidemic disease caused by the parasitism of Echinococcus multilocularis (Em) larvae in the intermediate or final host.

OBJECTIVE: To identify and analyze B-cell and T-cell (Th1, Th2, and Th17) epitopes of the Em antigen protein thrombospondin 3 (TSP3).

METHODS: The amino acid sequence of TSP3 was obtained, and the secondary structural characteristics of TSP3 were predicted using bioinformatics software to further predict its potential T-cell and B-cell epitopes. The spleen lymphocytes of $\mathrm{BALB} / \mathrm{c}$ mice, which were immunized with the TSP3 protein, were collected for co-culture with B-cell and T-cell antigen small peptides. The B-cell epitopes and T-cell epitope subtypes Th1, Th2, and Th17 were identified as having good immunogenicity. RESULTS: After identification, it was found that the predominant epitopes of B cells existing in TSP3 were T18-33, T45-55, and T110-122. Furthermore, the predominant epitopes of T cells existing in TSP3 were T33-42, T45-55, T80-90, and T110-122 in the T1 subtype, T45-55, T68-77, and T92-104 in the Th2 subtype, and T53-63 and T80-90 in the Th17 subtype.

CONCLUSIONS: Six T-cell and eight B-cell dominant epitopes of the TSP3 antigen were revealed; these results may be applied in the development of a dominant epitope vaccine.
\end{abstract}

Keywords: Echinococcus multilocularis, bioinformatics, epitopes

\section{Introduction}

Alveolar echinococcosis (AE) is an epidemic disease caused by the parasitism of Echinococcus multilocularis (Em) larvae in the intermediate or final host [1]. Hepatic AE exhibits an infiltrative growth in the host liver, which can cause liver injury, hepatic coma, and portal hypertension in patients [2]. In

\footnotetext{
${ }^{1}$ These authors contributed equally to this work.

${ }^{*}$ Corresponding author: Hai-Ning Fan, Department of Hepatopancreatobiliary Surgery, Affiliated Hospital of Qinghai University, 29 Tongren Road, Xining, Qinghai 810001, China. Tel.: +86 971 6162000; Fax: +86 971 6162000; E-mail: 2980948971@ qq.com.
}

0928-7329 (c) 2022 - The authors. Published by IOS Press. This is an Open Access article distributed under the terms of the Creative Commons Attribution-NonCommercial License (CC BY-NC 4.0). 
addition to the liver, AE can also occur in the brain $[3,4]$, which can seriously threaten a patient's life.

A host infected with $\mathrm{AE}$ will produce precise and complex immune responses to inhibit polypide growth and metastasis, as well as produce immune protection. The polypide will simultaneously develop a unique and effective immune escape [5].

At present, surgery with drug therapy is the preferred AE treatment choice. However, the surgical resection of this hydatid disease is often incomplete, causing patients to relapse easily [6]. Drug therapy has side effects, and its efficacy varies significantly among different patients [7]. Furthermore, other preventive measures have no distinct effects on AE. Hence, it is very important to determine a timely and effective approach to preventing hydatid infection.

Epitopes are a specific chemical group used for determining the specificity of antigens; they can be divided into T-cell and/or B-cell epitopes [8,9] and are capable of inducing an immune response against the invasion of AE. At present, epitope vaccines have been used for bacterial infection [10,11], viral infection, and tumors [12]. Hence, an epitope vaccine would be increasingly important for the future immune prevention of hydatid disease.

Determining antigen sites with good immunogenicity has been a bottleneck for immunogenic epitope vaccine research. In 2012, Dang et al. reported that the TSP family of Em surface antigens had good immunogenicity [13] and that the thrombospondin 3 (TSP3) protein had the best Em infection immunogenicity in the TSP family. These results suggest that preventing hydatid disease with a molecular vaccine is feasible.

The present study aims to predict and analyze the secondary structural characteristics of TSP3 and predict possible epitopes through the comprehensive comparison of different databases in accordance with specific published articles and with reference to the existing research database. The B-cell and T-cell (Th1, Th2, and Th17) epitopes will then be identified and analyzed, providing a theoretical basis for the development of multi-epitope anti-Em vaccines.

\section{Materials and methods}

\subsection{Antigen acquisition}

1. The TSP3 protein was provided by Dr. Tang Feng of the Medical College of Qinghai University.

2. The epitope small peptide was synthesized by Kingsy Biological Co., Ltd. (Nanjing, China) with a purity of $>95 \%$.

\subsection{Animals and experimental design}

Male SPF-level BALB/c mice (aged 7-8 weeks) with Experimental Animal License no. SCXK (Beijing) 2012-0001 were purchased from Weitong Lihua Experimental Animal Technology Co. Ltd. (Beijing, China).

\subsection{Experimental reagents and instruments (Tables 1 and 2)}

\subsubsection{Preparation of the reagents for the isolation and culture of mouse spleen lymphocytes}

The separation of spleen lymphocytes in mice was performed according to the reference of "Tianjin Hao 
Table 1

Experimental reagents

\begin{tabular}{lll}
\hline No. & \multicolumn{1}{c}{ Name } & \multicolumn{1}{c}{ Manufacturer } \\
\hline 1 & MTS & The United States Puluomaige company \\
2 & Mouse spleen lymphocyte separation & Tianjin Hao Yang, China \\
3 & FCS & Gibco, Australia \\
4 & RPMI-1640culture media & Sea Clone \\
5 & $70 \mu$ m Cell filter & BD, USA \\
6 & Bovine Serum Albumin (BSA) & Roche \\
7 & $10 \times$ PBS & Cable, Leybold, Beijing, China \\
8 & Cell culture 96 hole plate & Corelle, USA \\
9 & Tween-20 & DingGuochangsheng, Beijing, China \\
10 & TMB Substrate color & DingGuochangsheng, Beijing, China \\
11 & HRP labeled Goat anti-human IgG & Beijing Chinese fir \\
12 & Paraformaldehyde & Shanghai Biological Engineering, China \\
13 & Triton-100 & Sigma Aldrich, USA \\
14 & Flow tube & BD, USA \\
15 & PE-cy7 labeled anti-mouse IL-2 flow antibody & BD, USA \\
16 & PE-labeled anti-mouse IL-4 flow antibody & BD, USA \\
17 & Mouse IL-4, IL-2, IFN- $\gamma$, IL-17, TNF- $\beta$ ELISA Kit & Wuhan Youersheng, China \\
18 & Mouse IL-2, IL-4, IL-17A, IFN- $\gamma$ ELISpot Kit & AID, Germany \\
\hline
\end{tabular}

Table 2

Experimental instruments

\begin{tabular}{cll}
\hline No. & \multicolumn{1}{c}{ Name } & \multicolumn{1}{c}{ Manufacturer } \\
\hline 1 & Incubator & ChangzhouGuohua, China \\
2 & Mixing oscillator & Thermo Fisher, USA \\
3 & CO2 incubator & Thermo Fisher, USA \\
4 & Microplate reader & Bio-Rad, USA \\
5 & Electronic balance & Shanghai Min Bridge Instrument and Equipment Co., Ltd., China \\
6 & Clean bench & Suzhou Purification Equipment Factory, China \\
7 & Centrifuge & Thermo Fisher, USA \\
8 & Flow cytometer & Beckman Coulter, Inc., USA \\
\hline
\end{tabular}

Yang" regarding mouse spleen lymphocyte separation fluid. Phosphate buffered saline (PBS) preparation: Double distilled water was added to $100 \mathrm{~mL}$ of the PBS liquid $(10 \times)$ up to a volume of $1,000 \mathrm{~mL}$; the mixture was maintained at $4^{\circ} \mathrm{C}$ and heated at room temperature before use.

RPMI-1640 medium preparation: The base RPMI-1640 medium was prepared with $10 \%$ fetal bovine serum (FBS), $100 \mathrm{U} / \mathrm{ml}$ penicillin, and $100 \mathrm{~g} / \mathrm{ml}$ streptomycin. Antigen small-peptide solution preparation: The RPMI-1640 complete culture solution was added to the corresponding T-cell antigen peptide to obtain $14 \mathrm{ug} / \mathrm{ml}(2 \times$ storage solution $)$. All solutions were freshly prepared.

\subsubsection{The enzyme-linked immunosorbent assay detection reagent}

Coating buffer: Deionized water was added to the mixture, which comprised $1.6 \mathrm{~g}$ of $\mathrm{Na}_{2} \mathrm{CO}_{3}, 2.9 \mathrm{~g}$ of $\mathrm{NaHCO}_{3}$, and $0.2 \mathrm{~g}$ of $\mathrm{NaN}_{3}$, up to a volume of $1 \mathrm{~L}(\mathrm{pH}=9.6)$. Then, split charging was performed with sterile, clean vials, and the mixture was refrigerated and stored at $4^{\circ} \mathrm{C}$.

Washing liquor: The PBS was added to $1 \mathrm{ml}$ of Tween-20 up to a constant volume of 2,000 $\mathrm{ml}$.

Blocking Reagent: The $6 \mathrm{fg}$ of BSA, dissolved in $200 \mathrm{ml}$ of the washing buffer, was weighed and stored at $4^{\circ} \mathrm{C}$ after filtration.

Stop Buffer: A volume of $21.7 \mathrm{ml}$ concentrated sulfuric acid (1 H2SO4 M) was dropwise added to $178.3 \mathrm{~mL}$ of distilled water. All solutions were freshly prepared. 


\subsubsection{Flow detection reagent}

Fixed agent: A volume of $4 \mathrm{~g}$ of paraformaldehyde powder dissolved in $100 \mathrm{ml}$ was accurately weighed, and heating and magnetic stirring were slowly performed until the powder was completely dissolved. Then, the $\mathrm{pH}$ value was adjusted to 7.4 using sodium hydroxide tablets, and the mixture was filtered at 0.2 um.

Film breaking agent: The $0.1 \%$ Triton-100 solution was prepared with a Triton-100 solution and $0.1 \mathrm{mmol} / \mathrm{L}$ of PBS. The solution was freshly prepared.

Antibody dilution: A 98-mL PBS solution was added to $2 \mathrm{ml}$ of aseptic FBS. Washing liquid: A PBS solution containing $2 \%$ FBS.

\subsection{Experimental procedures}

\subsubsection{Mouse immunization}

A total of $50 \mathrm{SPF}$ BALB/c mice were randomly divided into two groups, i.e., the TSP3 (experimental) and the PBS (control) groups. Each mouse was administered with a 50- $\mu \mathrm{g}$ intraperitoneal injection. The vaccination was strengthened once a week and the mice were given Freund's adjuvant for immunity in all processes. At five days after the final immunization, the mice were sacrificed, and the spleen of each mouse was collected.

\subsubsection{Preparation of mouse spleen lymphocytes}

The mice were sacrificed after immunization and soaked with $75 \%$ disinfection alcohol for five minutes. Next, the abdominal cavity was cut, the spleen tissue was gradually collected, and dark and red mouse spleens were washed with sterile PBS three to five times and placed in sterile Petri dishes. The spleen single-cell suspension was prepared. The spleen lymphocytes obtained from the mice were separated according to the instruction "mouse spleen lymphocyte separation liquid", which was cultivated using an RPMI-1640 complete medium. After centrifugation, the bottom lymphocyte pellet was collected for flow cytometry.

\subsection{Prediction of the immunodominant epitopes of the Em TSP3 antigen}

First, the amino acid sequence of TSP3 was acquired from Genbank, and the secondary structural characteristics of the protein were predicted using the Self-optimized Prediction Method with Alignment (SOPMA) bioinformatics software (https://npsa-prabi.ibcp.fr/cgi-bin/npsa_autom-at.pl?page=/NPSA/np sa_sopma.html) [14]. The amino acid sequence of TSP3 obtained from GenBank was input into the software, and the four conformational states of the TSP3 protein secondary structure (helix, folding, corner, and curl) were, respectively, analyzed. The similarity threshold parameters were set to 8 , and the window width was set to 17 ; the remaining parameters were the default values.

The T-cell and B-cell antigen epitopes of TSP3 were further predicted using the online software immune epitope database (IEDB), SYFPEITHI, Bcepred, and ABCpred. Through the comprehensive analysis of different software, T-cell and B-cell dominant epitopes of the Em TSP3 antigenic protein were predicted. After clarifying the secondary structure of TSP3, the bioinformatics software IEDB (http://tools.immuneepitope.org/main/index.html) and SYFPEITHI (http://www.syfpeithi.de) [15] were used to predict and analyze potential T-cell antigen epitopes. The amino acid sequences of the TSP3 proteins were input into the software, and the IEDB parameters were adjusted as follows: "MHC allele (s)" was set to human leukocyte antigen (HLA)-A * 02:01; "length" was set to "all lengths"; the remaining parameters remained unchanged. The major histocompatibility complex (MHC)- 
I HLA-A*0201-restricted T-cell epitopes were predicted using the online prediction software SYFPEITHI, and the potential B-cell epitopes of TSP3 were analyzed using the Bcepred bioinformatics software (http://www.imtech.res.in/raghava/bcepred/bcepred_submission.html), as well as DABCpred (http://www.imtech.res.in/raghava/abcpred/) [16]. The amino acid sequences of TSP3 proteins were input into the software, and the parameter values of Bcepred were set as follows: hydrophilicity $=2$; flexibility $=1.9$; exposed surface area $=2.4$; antigenicity tendency $=1.8$. The remaining parameters remained unchanged. The epitope length of the ABCpred software was set to 10-16. The remaining parameters remain unchanged.

\subsection{Identification of Em TSP3 antigen B-cell immunodominant epitopes}

After obtaining a potential dominant B-cell epitope for TSP3, a small peptide was chemically synthesized. The enzyme-linked immunosorbent assay (ELISA) analysis of the serum obtained from patients with Em obtained the epitope with better immunogenicity. At the same time, the prokaryotic expression of TSP3 protein in the SPF BALB/c mice was immunized.

The spleen lymphocytes of the immunized mice were stimulated with chemically synthesized epitope peptides, and the proliferation was examined. Through ELISA reaction between a small peptide and patient serum, and the detection of spleen lymphocyte proliferation in mice, the comprehensive analysis identified B-cell epitopes with good antigenicity. The preparation of each reagent and specific experimental procedure was performed according to experimental instructions.

\subsection{Identification of Em TSP3 antigen T-cell immunodominant epitopes}

After obtaining the potential dominant T-cell epitope for TSP3, a small peptide was chemically synthesized. The ELISA analysis of serum obtained from patients with Em revealed the epitope with better immunogenicity. At the same time, the prokaryotic expression of the TSP3 protein was immunized in the SPF BALB/c mice.

The spleen lymphocytes of the immunized mice were stimulated with chemically synthesized epitope peptides. The ELISA, enzyme-linked immune absorbent spot (ELIspot) assay and flow cytometry were used to detect the expression of different cytokine types in the spleen lymphocytes stimulated by antigenic protein after epitope peptide stimulation. The antigen epitopes of the Thy, Th2, and Th17 types with good antigenicity were identified via comprehensive analysis. The preparation of each reagent and specific experimental procedure was performed according to experimental instructions.

\section{Results}

\subsection{Amino acid sequence of TSP3 protein}

According to GenBank records, the TSP3 protein consists of 148 amino acids (GenBank: ACJ02404.1), i.e., "mlktfavivaillvaeivcgivllvyrhefvgl Vgkemqreikdltahgrnasdpllksiyklqeeleccggvgptdwskpypasccks gkenctqpyqqgcavamyeqikdsslafgliilivcliqigavicacclakkvneyekv," which are coded by mRNA (GenBank: FJ384719.1) with a length of 646 bp.

\subsection{Prediction of the TSP3 protein secondary structure}

To evaluate the characteristics of the TSP3 antigen protein, the SOPMA software was used to predict 


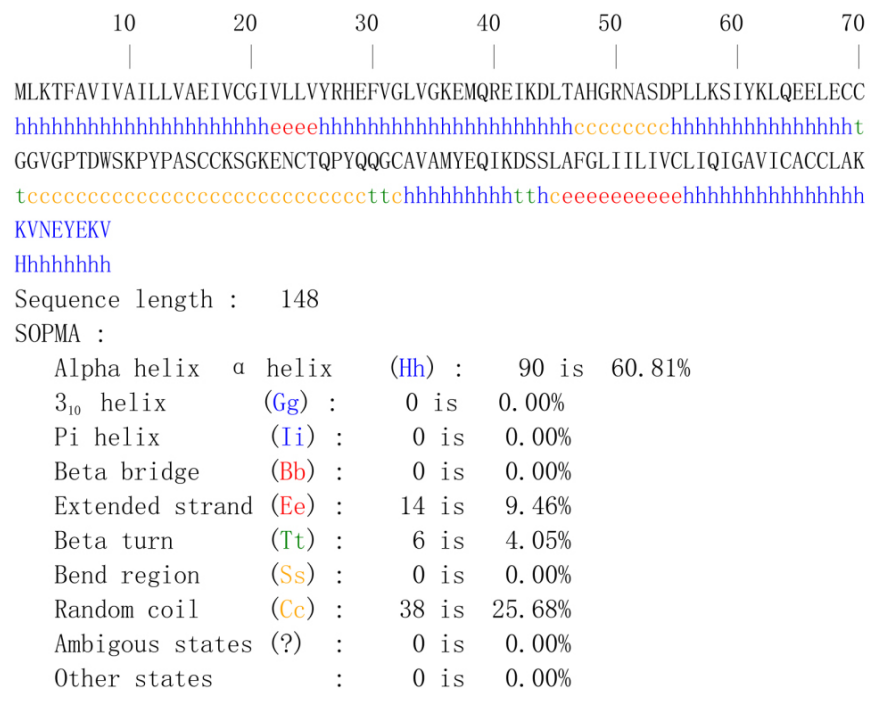
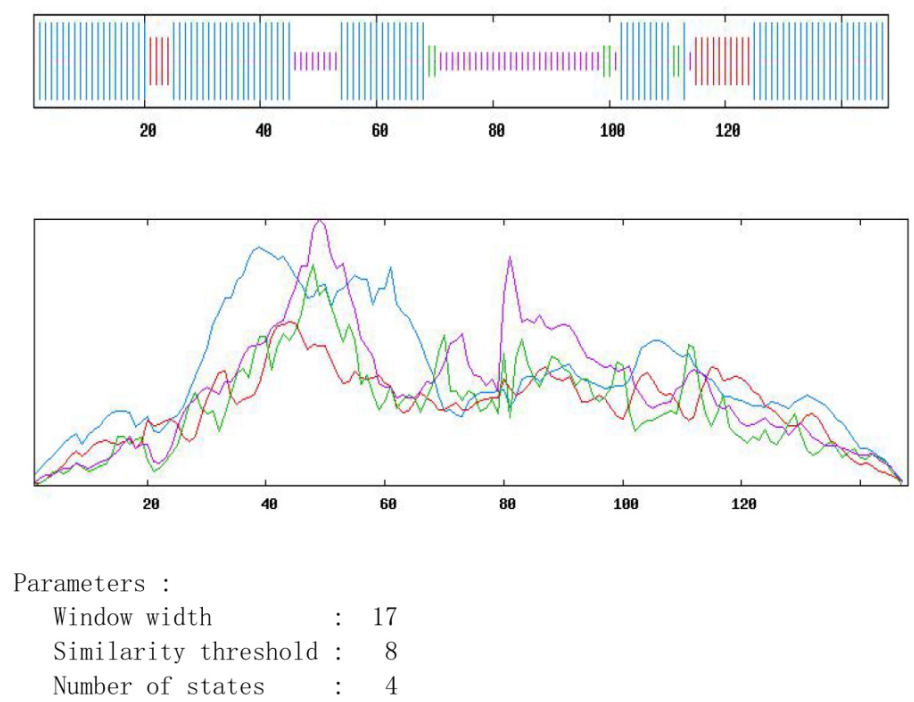

Fig. 1. Secondary structure prediction results of thrombospondin 3 protein. The lines in different colors represent different secondary structures as follows: blue $=\alpha$ helix; green $=\beta$ turn; red $=$ extended strand; purple $=$ random coil. $\mathrm{B}$ : The secondary structure prediction results for the thrombospondin 3 protein.

the protein's secondary structure (Fig. 1). In the extended protein chain ( $\beta$ folding), the random coil structure was the most likely potential dominant epitope. The results revealed that the TSP3 secondary structure of the random coil and the $\beta$ angle, $\alpha$ helix, and $\beta$-folding rates were $25.68 \%, 4.05 \%, 60.81 \%$, and $9.46 \%$, respectively.

\subsection{Prediction of TSP3 protein T-cell epitopes}

Determining the exact location of the epitope is very important for epitope vaccine development. In the present study, the restricted T-cell epitope of MHC-I HLA-A*0201 was predicted using the online 
Table 3

Analysis of thrombospondin 3 protein T-cell epitopes using the immune epitope database online prediction software

\begin{tabular}{lclcc}
\hline No. & Initiation site & Amino acid sequence & Length & Score \\
\hline 1 & 29 & EFVGLVGKEMQR & 12 & 100 \\
2 & 37 & EMQREIKDLTAH & 12 & 100 \\
3 & 53 & SDPLLKSIYKLQ & 12 & 100 \\
4 & 66 & ELECCGGVGPTD & 12 & 100 \\
5 & 81 & PYPASCCKSGKE & 12 & 100 \\
6 & 25 & VYRHEFVGLVGKE & 13 & 100 \\
7 & 29 & EFVGLVGKEMQRE & 13 & 100 \\
8 & 81 & PYPASCCKSGKEN & 13 & 100 \\
9 & 35 & GKEMQREIK & 9 & 100 \\
10 & 44 & DLTAHGRNASDP & 12 & 99 \\
11 & 85 & SCCKSGKENCTQ & 12 & 99 \\
12 & 53 & SDPLLKSIYKLQE & 13 & 99 \\
13 & 41 & EIKDLTAHGRNASD & 14 & 99 \\
14 & 53 & SDPLLKSIYKLQEE & 14 & 99 \\
15 & 81 & PYPASCCKSGKENC & 14 & 99 \\
\hline
\end{tabular}

Table 4

Analysis of thrombospondin 3 protein T-cell epitopes using the SYFPEITHI online prediction software

\begin{tabular}{lclcc}
\hline No. & Initiation site & Amino acid sequence & Length & Score \\
\hline 1 & 118 & GLIILIVCL & 9 & 30 \\
2 & 114 & PLAFGLIIL & 9 & 29 \\
3 & 55 & PLLKSIYKL & 9 & 26 \\
4 & 121 & ILIVCLIQI & 9 & 26 \\
5 & 12 & LLVAEIVCGI & 10 & 25 \\
6 & 114 & SLAFGLIILI & 10 & 25 \\
7 & 118 & GLIILIVCLI & 10 & 25 \\
8 & 9 & VAILLVAEI & 9 & 24 \\
9 & 59 & SIYKLQEEL & 9 & 24 \\
10 & 119 & LIILIVCLI & 9 & 24 \\
11 & 118 & GLIILIVCLI & 10 & 24 \\
12 & 115 & LAFGLIILIV & 10 & 24 \\
13 & 120 & IILIVCLIQI & 10 & 24 \\
\hline
\end{tabular}

software IEDB and SYFPEITHI. The scores of different sites were used to express the probability of the predicted T-cell epitope; the higher the site prediction score, the greater the area the epitope would likely have.

However, the two software solutions utilized different scoring systems; the high scores predicted by the IEDB software ranged from 99 to 100 (Table 3), while the high scores predicted by the SYFPEITHI software ranged from 24 to 30 (Table 4). Although the score systems of these two prediction software were different, they had a common aspect, i.e., higher scores could predict the potential epitope.

The results obtained through the IEDB software regarding the TSP3 T-cell epitopes showed that the T29-40, T37-48, T53-64, T66-77, and T81-92 epitopes had higher scores. However, the SYFPEITHI software predicted that the relative advantage of TSP3 epitopes was located in T118-126, T114-122, T55-63, and T121-129. When combining the prediction results of the two programs, eight potential T-cell dominant epitopes (T33-42, T45-55, T53-63, T68-77, T80-90, T92-104, T110-122, and T134-144) were finally identified. 


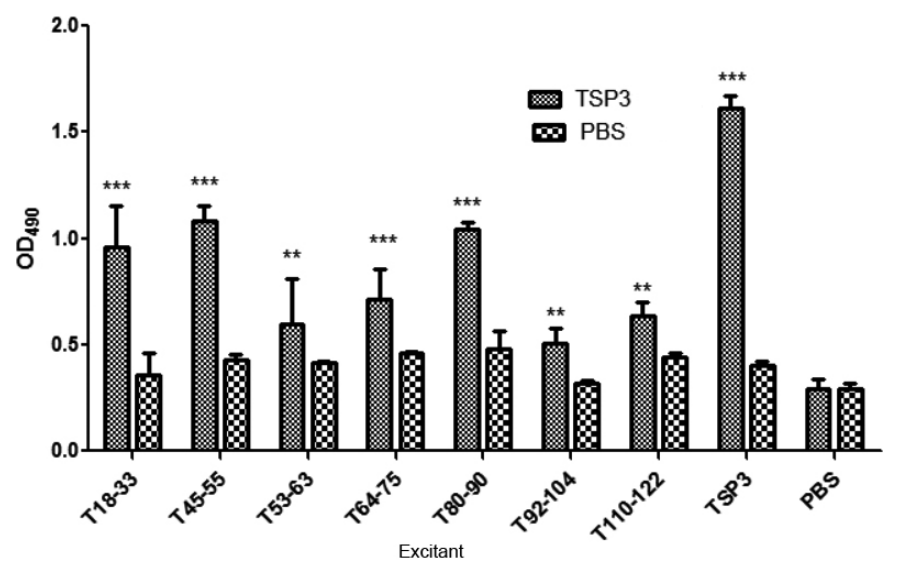

Fig. 2. The proliferation of specific B lymphocytes in mice immunized with thrombospondin $3 .{ }^{* * *} P<0.001$.

\subsection{The prediction of B-cell epitopes derived from the TSP3 antigen}

The Bcepred online software was used to predict the B-cell epitopes and analyze the hydrophilicity, elasticity, and surface antigen tendency, as well as the antigen exposure area of the TSP3 amino-acid sequence. The $\mathrm{ABCpred}$ online software was used to predict $\mathrm{B}$-cell epitopes with different epitope lengths $(10,12,14$, and 16).

Four regions of the TSP3 protein were predicted to be high hydrophilic regions: T35-41 (amino acid sequence: GKEMQRE), T48-55 (amino acid sequence: HGRNASDP), T85-97 (amino acid sequence: SCCKSGKENCTQP), and T108-114 (amino acid sequence: EQIKDSS). The two elastic regions were as follows: T46-52 (amino acid sequence: TAHGRNA) and T84-92 (amino acid sequence: ASCCKSGKE). The four possible antigenic regions were as follows: T16-36 (amino acid sequence: EIVCGIVLLVYRHEFVGLVGK), T55-75 (amino acid sequence: PLLKSIYKLQEELECCGGVGP), T117-130 (amino acid sequence: FGLIILIVCLIQIG), and T132-138 (amino acid sequence: VICACCL). The three exposed surface areas were as follows: T35-45 (amino acid sequence: GKEMQREIKDL), T62-68 (amino acid sequence: KLQEELE), and T139-148 (amino acid sequence: AKKVNEYEKV).

The TSP3 B-cell epitopes were predicted using the ABCpred online software for accurate prediction, and it was revealed that T59-73, T39-54, T80-95, T74-87, and T129-144 had higher scores. According to the secondary structural characteristics of the TSP3 protein, the predicted seven potential B-cell epitopes were T18-33, T45-55, T53-63, T64-75, T80-90, T92-104, and T110-122.

\subsection{Lymphocyte proliferation assay detection}

As shown in Fig. 2, the mouse spleen lymphocytes immunized with TSP3 protein produced lymphocyte proliferation under stimulation with B-cell small antigen peptide, TSP3 antigen protein, and PBS; there were differences in proliferation, and the PBS immune group had no lymphocyte proliferation upon receiving the above antigen stimulation.

These results indicate that the B-cell antigen peptide had antigenicity and that there were differences in antigenicity (lower in the B-cell peptide than in TSP3 but higher in the B-cell peptide than in PBS lymphocyte stimulation). According to the effect of the antigen peptide on lymph cell proliferation, the following dominant epitopes were selected: T18-33, T45-55, and T110-122. 


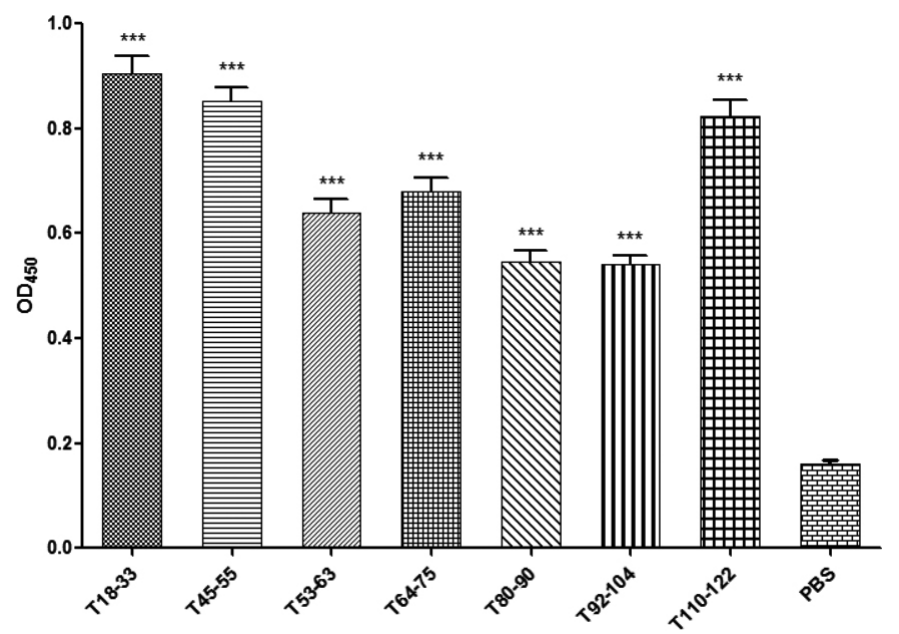

Fig. 3. The enzyme-linked immunosorbent assay test on thrombospondin 3 B-cell antigen peptide acting with the serum, which was obtained from patients with alveolar echinococcosis. ${ }^{* * *} P<0.001$.

\subsection{Detection of the reaction between the TSP3 B-cell antigen and the patient's serum using the ELISA method}

The artificial antigen peptide was packed with ELISA plates, and the antigen epitope was screened with anti-Em serum obtained from patients with AE (Fig. 3). The artificial TSP3 B-cell antigen small peptide could produce an ELISA-specific reaction with the AE serum, and the absorbance was significantly higher in the experimental group than in the PBS control group $(P<0.001)$; this indicated that the $\mathrm{B}$ antigen peptide had antigenicity.

The absorbance of the TSP3 antigen peptide was different, indicating that the antigenicity of the B epitope varied, and the antigenic small peptide (T18-33, T45-55, and T110-122) was closely combined with the serum antibody of patients with AE. Furthermore, the optical density value was higher compared with other small peptides $(P<0.01)$. Hence, it was concluded that T18-33, T45-55, and T110-122 were the dominant B-cell epitopes.

\subsection{Screening of the Tepitopes with different cytokines using the ELISA method}

After the spleen-cell stimulation with the T-epitope peptide, the supernatants were collected, and cytokines interleukin (IL)-2 (Fig. 4A), tumor necrosis factor (TNF)- $\gamma$ (Fig. 4B), TNF- $\beta$ (Fig. 4C), IL-4 (Fig. 4D), and IL-17 (Fig. 4E) were detected. The Th1 cytokines were detected in each culture supernatant and revealed that different stimuli (T-cell antigen peptide and TSP3 antigen protein) could stimulate lymphocytes sensitized by TSP3 secreting IL-2, interferon (IFN)- $\gamma$, and TNF- $\beta$. However, the difference in PBS immune cells that secreted cytokines among the cytokines was not statistically significant.

Furthermore, T110-122, T134-144, T33-42, and T80-90 could increase IL-2 expression; T45-55, T33-42, T80-90, and T110-122 could increase IFN- $\gamma$ expression, and T45-55, T80-90, T92-104, and T33-42 could increase TNF- $\beta$ expression. This indicated that T33-42, T45-55, T80-90, and T110-122 were the advantages of the Th1 epitope of TSP3.

Each group of IL-4 ELISA absorbance values was different in terms of lymphocyte supernatant, indicating that different antigen peptides had different effects on lymphocyte IL-4 expression. The antigen 

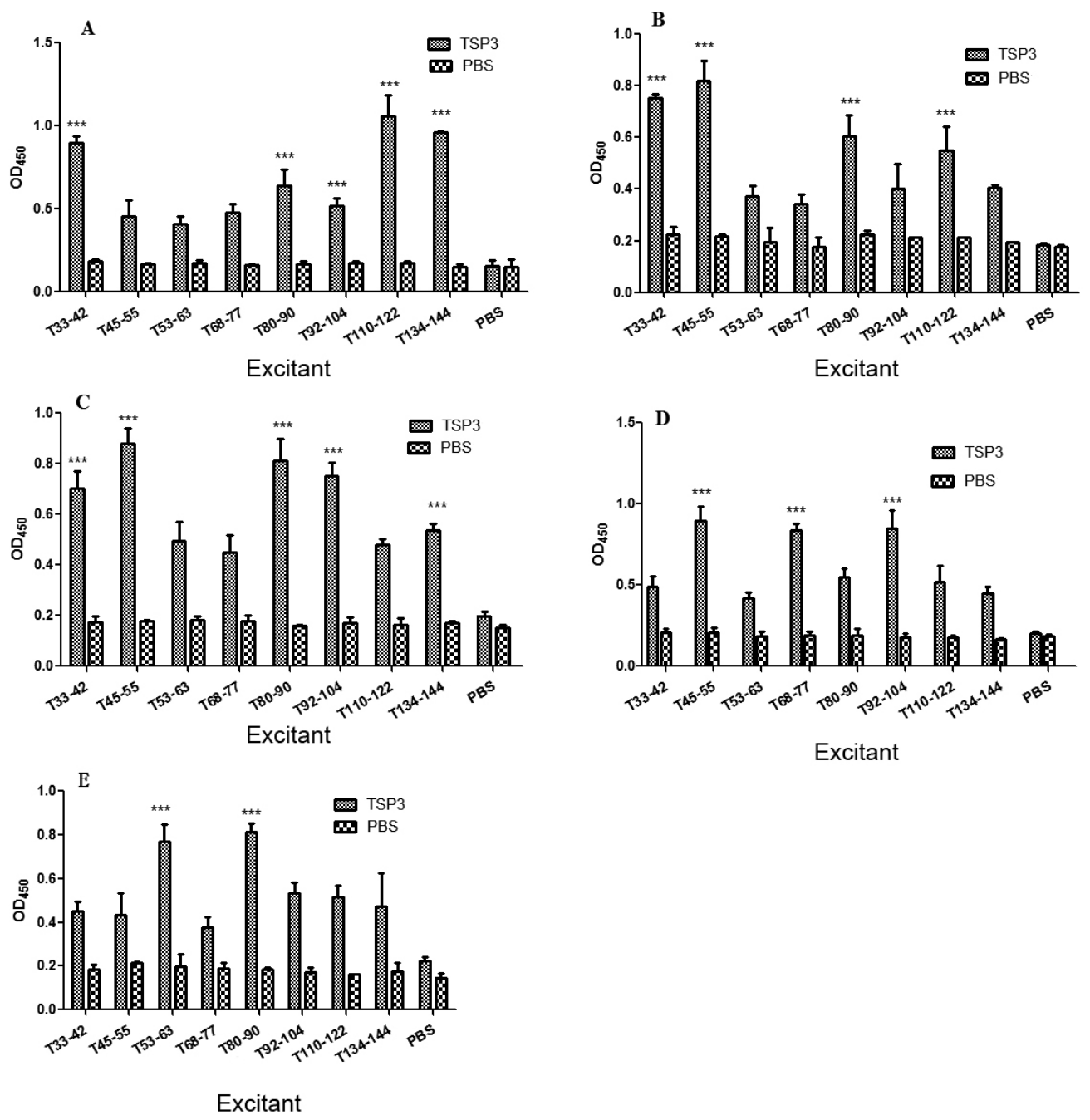

Fig. 4. The enzyme-linked immunosorbent assay test on the cytokines of mouse spleen lymphocytes immunized with thrombospondin 3. A: The interleukin-2 test; B: the interferon-y test; C: the tumor necrosis factor- $\beta$ test; D: the interleukin-4 test; E: the interleukin- 17 test. $^{* * *} P<0.001$.

peptides that could induce the high expression of IL-4 were T45-55, T68-77, and T92-104. Hence, T45-55, T68-77, and T92-104 were selected as the dominant Th2 epitope of TSP3.

Each group of IL-17 ELISA absorbance values was different in terms of lymphocyte supernatant, indicating that different antigen peptides had different effects on lymphocyte IL-17 expression. The antigen peptides that could induce a high expression of IL-17 were T53-60 and T80-90. Hence, T53-60 and T80-90 were selected as the dominant Th17 epitopes of TSP3.

\subsection{Screening of T epitopes with different cytokines using ELISpot}

Spleen lymphocytes obtained from the mice sensitized by TSP3 and PBS were stimulated with the advantage of epitope peptides after the preliminary ELISA screening. The ELISpot test results regarding cytokines are presented in Fig. 5. 

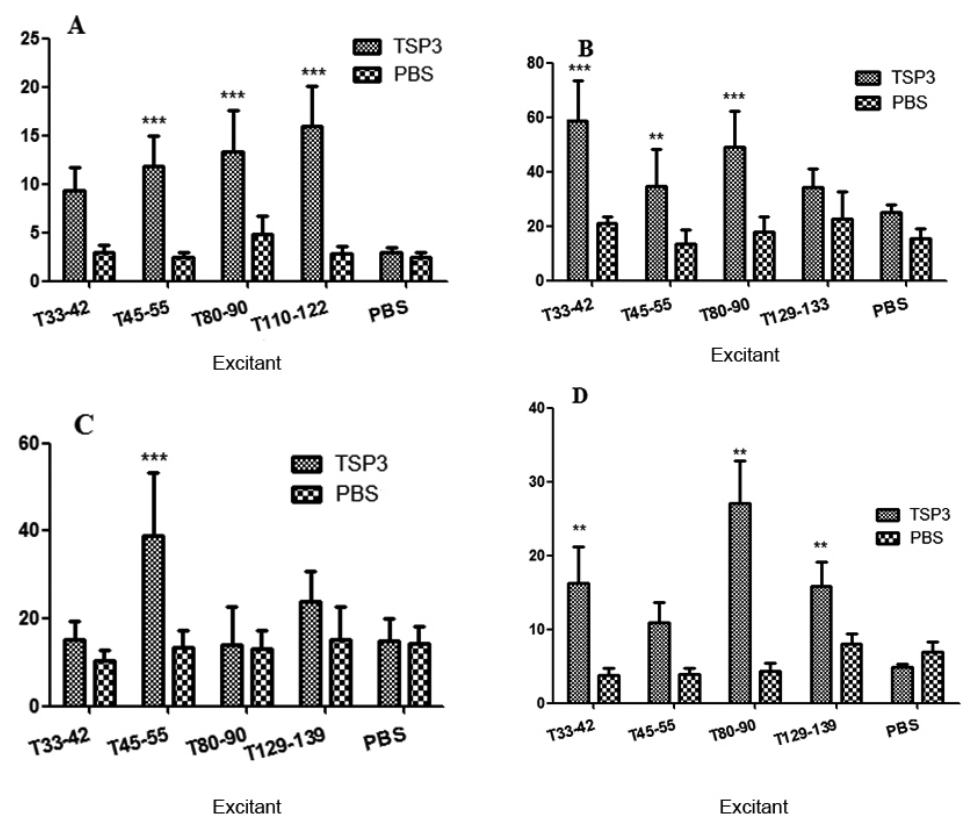

Fig. 5. The enzyme-linked immune absorbent spot test of the cytokines of mouse spleen lymphocytes immunized with thrombospondin 3. A: The interleukin-2 test; B: the interferon- $\gamma$ test; $\mathrm{C}$ : the tumor necrosis factor- $\beta$ test; D: the interleukin-4 test. ${ }^{* * *} P<0.001$

Each dominant antigen peptide could stimulate a high expression of IL-2 and IFN- $\gamma$. It was concluded from these results that T33-42, T45-55, T80-90, and T110-122 were the advantages of the Th1 epitope of TSP3; these results were the same as those of the cytokine ELISA test (Fig. 5A and B).

The effect of the advantage of the Th1 antigen peptide lymphocytes stimulated the expression of IL-4, which was different; there were also obvious differences between the TSP3 and the PBS immune groups (Fig. 5C). Furthermore, the expression of IL-4 was significantly higher in the small peptide T45-55 antigen stimulation group than in the other groups $(P<0.01)$.

It was concluded from the ELISpot assay results that T45-55 was the advantageous Th2 antigen peptide of TSP3. There were differences in the lymphocyte supernatant among all groups of IL-17A ELISA absorbance values (Fig. 5D), indicating that different antigen peptides had different effects on lymphocyte IL-17A expression. The antigen peptide that could induce a high expression of IL-17A was T80-90. Hence, T80-90 was selected as the dominant Th17 epitope of TSP3.

\subsection{Verification of dominant epitopes using flow cytometry}

The expressions of cytokines IL-2 and IL-4 after sensitization were detected using flow cytometry to again confirm the identification of the Th1 epitopes of the antigen. After the lymphocyte stimulation with the small peptide, there was a significant difference in positive cells between cytokines IL-2 and IL-4 $(P<0.05)$, and the expression of the IL-2 lymphocyte ratio was significantly higher than the expression of IL-4. The detection results are presented in Fig. 6.

The T33-42 ratio for the induction of IL-2 lymphocyte secretion was approximately $7.58 \%-9.91 \%$, and the percentage of lymphocytes with a positive expression of IL- 4 was approximately $3.01 \%-8.78 \%$. The T80-89 ratio for the induction of IL-2 lymphocyte secretion was approximately $12.47 \%-13.08 \%$, and the percentage of lymphocytes that had a positive expression of IL-4 was approximately $6.84 \%-4.17 \%$. The 
A

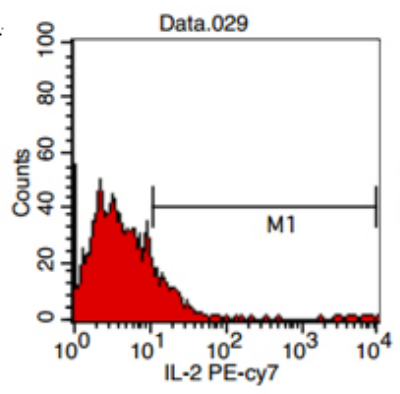

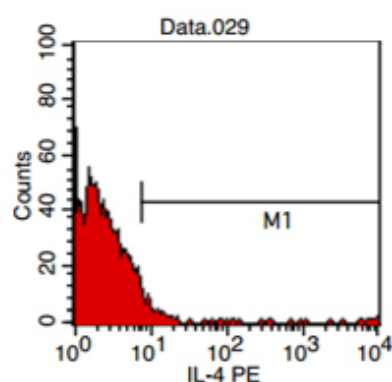

B
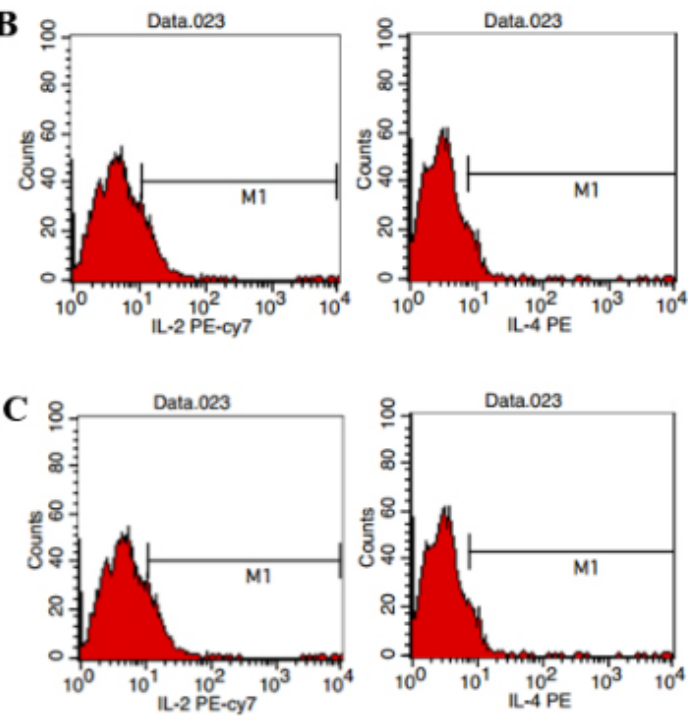

Fig. 6. The Fcw test of the cytokines of mouse spleen lymphocytes immunized with thrombospondin 3. A: The E7-13 test; B: the E80-89 test; C: the E129-139 test.

T110-122 ratio for the induction of IL-2 lymphocyte secretion was $10.92 \%-12.46 \%$, and the lymphocyte percentage for the positive expression of IL-4 was approximately $2.96 \%-6.81 \%$.

\section{Discussion}

In the long-term "immune dialogue" process between Em and the host, Em also produces "immune" to the host. After infecting the host, the Em antigen material and excretion can be immediately identified by the host's immune system, which, in turn, generates an immune response to identify, inhibit, and clear the parasites. At the same time, the parasites can also resist host immune damage through immune escape. Therefore, the analysis of specific antigen epitopes of multilocular antigen proteins can help in preparing effective epitope-based vaccines for parasites when conducting specific immune prevention.

An epitope is a specific chemical group that can be used to determine the specificity of antigen protein molecules. Ma et al. [17] regarded the $A g B 1$ gene sequence of Echinococcus granulosus as the foundation and predicted four B-cell antigen epitopes and four T-cell antigen epitopes. Li et al. [18] regarded the EB virus nucleoprotein-1 (EBNA 1) gene sequence as the foundation and predicted EBNA-1 B-cell epitopes, analyzed the membrane structure domain and other parameters (e.g., hydrophilicity), and compared it with the sequences of human self-associated antigens. 
Protein secondary structures are closely correlated with epitope distribution. Furthermore, the $\alpha$-helix and $\beta$-sheet, which are present in the interior of the protein, play important roles in maintaining the stability of the protein secondary structure; however, they do not easily combine with antigen molecules [19].

In the present study, the proportions of the $\alpha$-helix and $\beta$-sheet in TSP3 were $60.81 \%$ and $9.46 \%$, respectively, indicating that the TSP 3 antigen had good stability. The random curl and $\beta$-turn accounted for $25.68 \%$ and $4.05 \%$ of the protein, respectively.

TSP3 has six potential antigenic regions; among these, the random coil region had a higher proportion of T47-54 and T72-99, indicating that these two regions were strongly antigenic.

Using the IEDB and SYFPEITHI online software for analysis, it was predicted that eight T-cell epitopes of the TSP3 protein were located at T33-42, T45-55, T53-63, T68-77, T80-90, T92-104, T110-122, and T134-144. The Bcepred and ABCpred online software were used to comprehensively analyze the epitope parameters of TSP3 and predict and analyze the potential predominant epitopes of seven TSP3 B-cells (T18-33, T45-55, T53-63, T64-75, T80-90, T92-104, and T110-122).

TSP3 was observed to have five epitopes with B/T dual cellularity (T/V-55, T53-63, T80-90, T92-104, and T110-122). The comprehensive predictions of different software revealed that the peptide with a higher score was the potential dominant epitope; they then found the appropriate antigen site and amino acid sequence of antigen peptide for the subsequent development of a multi-epitope vaccine.

The present study clarified the bioinformatic characteristics of the TSP3 protein, thereby providing a basis for the identification and screening of dominant epitopes and providing a theoretical foundation for the development of epitope vaccines for immunoprophylaxis and the treatment of Em.

The bioinformatics prediction and identification of B-cell epitopes have been widely used in antigenic proteins such as Em. However, only dominant epitopes are induced. When the protective immune response is absolutely dominant, it can stimulate optimal immune protection for the host.

The spleen is the largest lymphoid organ in the human body; the proportion of the total number of lymphocytes in spleen T-cells is 35\%-50\%, and the proportion of B-cells is as high as 50\%-65\% [20]. After antigen stimulation, B-cells would proliferate to form germinal centers, produce immunoglobulin $\mathrm{M}$ and immunoglobulin $\mathrm{G}$, and exert humoral immunity. Therefore, the proliferation of lymphocytes can reflect B-cell antigens; the higher the degree of proliferation of lymphocytes stimulated by small peptides, the stronger the antigens of the small peptides and the greater the possibility of them becoming dominant epitopes.

At present, the ELISA assay is widely used in the detection of other parasitic infections [21] and the identification of epitopes [22]. In the present study, lymphocyte proliferation reaction was monitored, and the specific ELISA reaction was used with the serum obtained from patients with AE to identify the B-cell antigen epitopes and predict the TSP3 bioinformatics of Em antigens. It was also used to determine the advantageous TSP3 B-cell antigen epitopes (T18-33, T45-55, and T110-122).

The MHC-I molecules are distributed on the surface of all nucleated cells, while MHC-II molecules are distributed on the surface of antigen-presenting and activated T cells. As it is unclear what kind of immune response will be induced by the predicted small peptide of the $\mathrm{T}$ antigen, a method with a wider range of relative immunogenicity was selected in this study.

Studies $[23,24]$ confirmed that Th1 cytokines (e.g., IFN- $\gamma$ ) and Th2 cytokines (e.g., IL-4) play important roles in various immunopathological processes, such as echinococcosis and malaria. Researchers $[25,26]$ have continuously raised mice inoculated with original lice and found that the expression level of Th1 cytokines was significantly reduced, while the opposite Th2 cytokine level was significantly increased. This indicated that the Th2 immune responses were dominant. This can reduce the body's protective immunity against killing the original head lice. 
After the successful treatment of patients with hydatid disease, the expression of Th2-type cytokines IL-10 was reduced, and IL-4 was not detected; meanwhile, the Th1- type cytokine IFN- $\gamma$ was expressed at high levels. Patients with hydatid infection exhibited a contrasting result.

The above studies indicate that Th1 cell immunity plays a major role in the host of hydatidosis in the stable phase and that Th2 cell immunity plays an important role in the excessive and active phases.

The immune escape mechanism of Em may be achieved through the balance of Th1 and Th2 cytokines. In the present study, it was found that after the statistical analysis of the production of Th1 cytokines (IL-2, IFN- $\gamma$, and TNF- $\beta$ ) induced by T-cell epitopes, T33-42, T45-55, T80-90, and T110-122 were the Th1 cell advantageous epitopes of TSP3. The Th1 lymphocytes could specifically bind to Th1 epitopes and secrete cytokines such as IL-2 after activation and inhibit the differentiation and function of Th2 cells; this resulted in a defensive immunoprotective response against Em.

The anti-multifamily Echinococcus vaccine induces a Th1-type immune response in the host and enhances the host's immunity against Em infection. Therefore, the development of a multi-epitope antiechinococcal vaccination against Th1-type epitopes can reduce the prevalence of hydatidosis infection.

Additionally, T45-55, T68-77, and T92-104 were the Th2 dominant epitopes of TSP3 according to the statistical analysis of Th2 cytokine production by each T-cell epitope. The Th2-type epitopes could induce a Th2-type immune response in the host and inhibit the differentiation and function of Th1 cells, leading to the escape of the vesicles from the host's immune system and favoring the larvae of Em in the host. Therefore, removing Th2 epitopes can increase the ability of epitope vaccines to induce immune protection.

\section{Conclusion}

After analyzing the T17 cytokine production induced by each T-cell epitope, T53-63 and T80-90 were found to be the Th17 dominant epitopes of TSP3. The Th17 cells were involved in innate immune and adaptive immune responses, played an important role in the host's immune defenses and inflammatory injury, and participated in the early immune clearance of parasitic infection and the immune effects of the late adaptive immune response. However, in patients with a hydatid infection, there was an imbalance in the Thl7/Treg immune response, and the immunosuppressive immune response was predominant. Therefore, the multi-epitope vaccine against multi-epidermal echinococcus with the Th17 epitope can be used for the treatment of patients with an echinococcal infection. However, this approach requires additional research.

\section{Acknowledgments}

None to report.

\section{Author contributions}

All authors have contributed significantly to the manuscript and declare that the work is original and has not been submitted or published elsewhere.

\section{Conflict of interest}

None of the authors have any financial disclosure or conflict of interest. 


\section{Funding}

This study was supported by the Qinghai Science and Technology Department (No. 2014-ZJ-719).

\section{References}

[1] Craig PS. Echinococcosis Working Group in China. Epidemiology of human alveolar echinococcosis in China. Parasitol Int. 2006; 55(Suppl): S221-5. doi: 10.1016/j.parint.2005.11.034.

[2] Grosso G, Gruttadauria S, Biondi A, et al. Worldwide epidemiology of liver hydatidosis including the Mediterranean area. World J Gastroenterol. 2012; 18(13): 1425-37. doi: 10.3748/wjg.v18.i13.1425.

[3] Debourgogne A, Goehringer F, Umhang G, et al. Primary cerebral alveolar echinococcosis: Mycology to the rescue. J Clin Microbiol. 2014; 52(2): 692-4. doi: 10.1128/JCM.02843-13.

[4] Atalan G, Sivrioglu AK, Sönmez G, et al. A case of alveolar echinococcosis presenting as cerebral and spinal intradural metastases. Eurasian J Med. 2016; 48(2): 149-52. doi: 10.5152/eurasianjmed.2015.15003.

[5] Siracusano A, Delunardo F, Teggi A, et al. Cystic echinococcosis: Aspects of immune response, immunopathogenesis and immune evasion from the human host. Endocr Metab Immune Disord Drug Targets. 2012; 12(1): 16-23. doi: $10.2174 / 187153012799279117$.

[6] Chen KF, Tang YY, Wang R, et al. The choose of different surgical therapies of hepatic alveolar echinococcosis: A singlecenter retrospective case-control study. Medicine (Baltimore). 2018; 97(8): e0033. doi: 10.1097/MD.0000000000010033.

[7] Wen-Jun Z, Xiu-Min H, Ya-Min G. Progress in researches of benzimidazole in treatment of echinococcosis. Zhongguo Xue Xi Chong Bing Fang Zhi Za Zhi. 2017; 29(4): 530-533. Chinese. doi: 10.16250/j.32.1374.2017057.

[8] Almeida RR, Rosa DS, Ribeiro SP, et al. Broad and cross-clade CD4+ T-cell responses elicited by a DNA vaccine encoding highly conserved and promiscuous HIV-1 M-group consensus peptides. PLoS One. 2012; 7(9): e45267. doi: 10.1371/journal.pone.0045267.

[9] Zhang Z, Chen J, Shi H, et al. Identification of a conserved linear B-cell epitope in the M protein of porcine epidemic diarrhea virus. Virol J. 2012; 9: 225. doi: 10.1186/1743-422X-9-225.

[10] Li HB, Zhang JY, He YF, et al. Systemic immunization with an epitope-based vaccine elicits a Th1-biased response and provides protection against Helicobacter pylori in mice. Vaccine. 2012; 31(1): 120-6. doi: 10.1016/j.vaccine.2012.10.091.

[11] Mollica A, Stefanucci A, Costante R. Strategies for developing tuberculosis vaccines: Emerging approaches. Curr Drug Targets. 2013; 14(9): 938-51. doi: 10.2174/1389450111314090002.

[12] Hazama S, Maeda K, Oka M. Epitope peptide vaccine with oncoantigen for cancer and its biomarker. Nihon Rinsho. 2012; 70(12): 2189-93. Japanese.

[13] Dang Z, Yagi K, Oku Y, et al. A pilot study on developing mucosal vaccine against alveolar echinococcosis (AE) using recombinant tetraspanin 3: Vaccine efficacy and immunology. PLoS Negl Trop Dis. 2012; 6(3): e1570. doi: 10.1371/journal.pntd.0001570.

[14] Geourjon C, Deléage G. SOPMA: Significant improvements in protein secondary structure prediction by consensus prediction from multiple alignments. Comput Appl Biosci. 1995; 11(6): 681-4. doi: 10.1093/bioinformatics/11.6.681.

[15] Vaughan K, Peters B, Larche M, et al. Strategies to query and display allergy-derived epitope data from the immune epitope database. Int Arch Allergy Immunol. 2013; 160(4): 334-45. doi: 10.1159/000343880.

[16] Sollner J, Grohmann R, Rapberger R, et al. Analysis and prediction of protective continuous B-cell epitopes on pathogen proteins. Immunome Res. 2008; 4: 1. doi: 10.1186/1745-7580-4-1.

[17] Ma XM, Hu XA, Tuerxun Arzgul, et al. Bioinformatics prediction of AgB1 antigen epitopes of echinococcus granulosus. Science \& Technology Review. 2013; 31(27): 27-30. Chinese. doi: 10.3981/j.issn.1000-7857.2013.27.003.

[18] Li L, Zhu S, Li W, et al. Prediction and research on homology of B-cell epitopes of Epstein-Barr virus nuclear antigen-1. Sheng Wu Yi Xue Gong Cheng Xue Za Zhi. 2011; 28(2): 371-5. Chinese.

[19] Ma FS, Zhang L, Wang Y, et al. Advance on prediction methods of B-cell antigen epitope. China Animal Husbandry and Veterinary Medicine. 2016; 43(1): 63-67. Chinese. doi: 10.16431/j.cnki.1671-7236.2016.01.010.

[20] Li ZF, Zhang S. The progress and prospect of fundamental research of the spleen. Journal of Xi' an JiaoTong University (Medical Science). 2008; 29(1): 1-6. Chinese.

[21] Yuan Y, Ma Y, Wang Y, et al. Clinical application of ELISA kit in detection of anti-Cysticercus IgG antibody. China Tropical Medicine. 2015; 15(10): 1257-1259. Chinese. doi: 10.13604/j.cnki.46-1064/r.2015.10.31.

[22] Jiang L, Feng Z, Zhang YG, et al. Serodiagnosis of the recombinant multi-epitope antigens from antigen B subunits of Echinococcus granulosus. Zhongguo Ji Sheng Chong Xue Yu Ji Sheng Chong Bing Za Zhi. 2013; 31(6): 438-42. Chinese.

[23] Gu ML, Li YM, Zheng H. Research progress on the role of IFN- $\gamma$ and IL-4 in parasite infection immunity. Endemic Diseases Bulletin. 2010; 25(3): 69-71. Chinese. 
[24] Yang M, Li WG. Changes of cytokines in mice immunized with recombinant Bb-Em II/3-Em14-3-3 vaccine of Echinococcus multilocularis. Chinese Journal of Cellular and Molecular Immunology. 2008; 24(8): 781-784. Chinese. doi: 10.3321/j.issn:1007-87382008.08.010.

[25] Maccario R, Podestà M, Moretta A, et al. Interaction of human mesenchymal stem cells with cells involved in alloantigenspecific immune response favors the differentiation of CD4+ T-cell subsets expressing a regulatory/suppressive phenotype. Haematologica. 2005; 90(4): 516-25.

[26] Casiraghi F, Azzollini N, Cassis P, et al. Pretransplant infusion of mesenchymal stem cells prolongs the survival of a semiallogeneic heart transplant through the generation of regulatory T cells. J Immunol. 2008; 181(6): 3933-46. doi: 10.4049/jimmunol.181.6.3933. 